\title{
Modelling of corona discharge in cylinder-wire-plate electrode configuration
}

\author{
Laurentiu Marius Dumitran ${ }^{\mathrm{a}}$, Lucian Dascalescu ${ }^{\mathrm{b}, *}$, Petru V. Notingher ${ }^{\mathrm{a}}$, Pierre Atten ${ }^{\mathrm{c}}$ \\ ${ }^{a}$ Laboratory of Electrical Materials, University of Bucarest, Splaiul Independentei, N. 313, 060042 Bucharest, Romania \\ ${ }^{\mathrm{b}}$ Equipe Electronique et Electrostatique, LAII-ESIP, UPRES-EA 1219, 4 avenue de Varsovie, 16021 Angoulême Cedex, France \\ ${ }^{\mathrm{c}}$ LEMD-CNRS, 25 avenue des Martyrs, B.P. 166, 38042 Grenoble Cedex 9, France
}

\begin{abstract}
Numerical computation of the electric field strength and ionic space charge density in electrode systems consisting of ionizing wire and non-ionizing cylinder, connected to the same DC high-voltage supply and facing a grounded plate, is a difficult problem, which is of interest to several electrostatic processes applications. In a previous study a simple numerical method has been proposed to calculate the spatial distributions of electric field and ionic space charge in a case of a continuum and uniform corona discharge originating at the surface of the wire. The aim of the present paper is to improve the physical model of the corona discharge in this particular electrode configuration, by assuming a more realistic law of charge injection on the wire circumference. The computations were carried out for an ionizing wire of radius $r=0.1 \mathrm{~mm}$, located at different distances $h$ from a metallic tubular support of radius $R=13.4$ mm.The initial conditions of the corona discharge took into account the non-uniformity of the charge injection around the ionizing wire electrode. The computational results were compared with those obtained under the assumption of uniform corona discharge. The comparison pointed out that neither the non-uniformity of the electric field nor that of the charge injection can be neglected. They depend on the geometry of the electrode system and affect the distribution of the electric field and of the space charge density in the inter-electrode gap.
\end{abstract}

Keywords: Corona discharge; Dual ionizing electrode; Electric field and space charge computation

\section{Introduction}

The corona discharge is used in many electrostatic processes [1]. In a large group of applications, such as the separators of mixed granular solids, the precipitators of dust, and the powder coating systems, the corona discharge is used to electrically charge small insulating particles, in view of their processing in a high-intensity electric field. In other devices, the non-thermal plasma generated by corona discharge promotes the chemical decomposition of some toxic gases [2].

The corona (or "ionizing") electrodes typically consist of needles, wires or blades, connected to high-voltage supplies. In some cases (electrostatic separators), the ionizing electrodes have a more elaborate shape: the

\footnotetext{
${ }^{*}$ Corresponding author.

E-mail address: ldascalescu@iutang.univ-poitiers.fr (L. Dascalescu).
}

"barbed electrode" that consists of a metallic cylinder with ionizing points, spiral, ribbon peak, ribbon square teeth, serrated strip, etc. [3].

For any shape or type of ionizing electrode, the corona discharge is characterized by two regions: a thin layer, very close to the active electrode surface called "ionization zone" that involves such physical process as ionization, attachment and recombination, and a "drift zone" where the ions are driven by the field toward a collecting electrode. The complete physical model of the corona discharge is rather complicated. However, many authors proposed numerical models that take into account the phenomena occurring in the very thin ionizing zone [4].

Considering the ionizing thin layer only as a "source" of mono-polar ions, the physical phenomena specific to this region can be neglected and the model of corona discharge can be drastically simplified. In this case, the study of corona discharge is reduced to the evaluation of the space 
charge density due to the mono-polar ions that move with constant mobility along the field lines. This approach is generally suitable to model the macroscopic aspect of the corona electric field, as affected by the ionic space charge. For simple two electrode configurations like needle-plate or wire-plate, this problem can be readily solved using various techniques such as finite differences FDM [5], finite element FEM [6,7], the combined method of finite element, boundary element BEM and the method of characteristics MOC $[8,9]$, as well as the charge simulation method CSM [10]. However, the implementation of these methods in the case of ionizing electrodes associated with non-ionizing electrodes at the same or a different potential (Fig. 1) is not straightforward. The existence of a singular point of zero electric field in the region located between the ionizing and non-ionizing elements of the dual electrode requires a very complicated treatment of the governing field-charge equations.

In a recent paper [11] a simple method based on conformal mapping was employed to investigate the spatial distribution of the electric field and the ionic charge in the geometry showed in Fig. 1. The aim of the present study is to analyze and refine the physical model adopted in [11]. In this purpose, the authors propose to replace the assumption that the charge injection (corona discharge) is uniform around the ionizing wire with a more realistic non-uniform

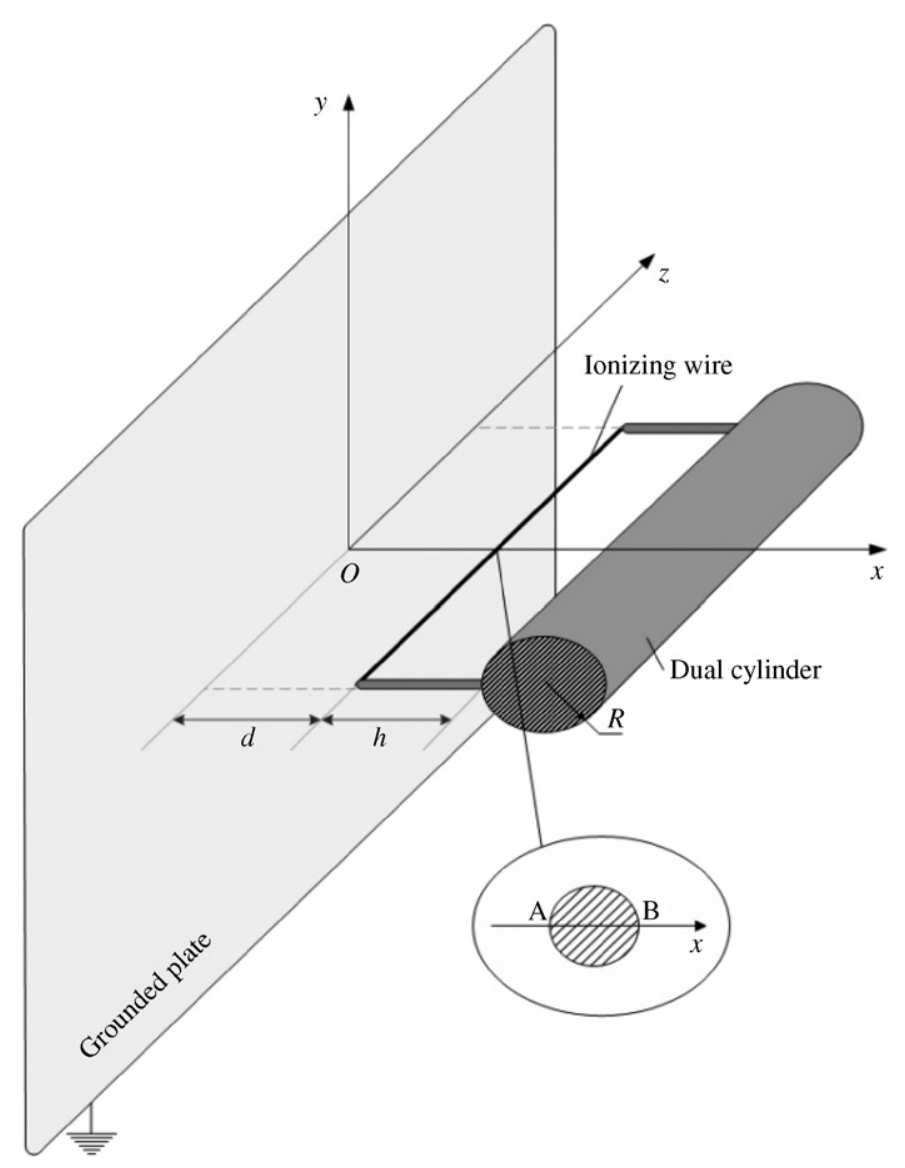

Fig. 1. Schematic view of the electrode system under study. charge injection law that takes into account the field modulation at the wire surface.

\section{Physical and numerical modelling of the corona discharge}

\subsection{Physical model}

The physical model of the corona discharge is exposed in relation with the electrode configuration schematized in Fig. 1. The ionizing wire and the electrostatic cylinder are at the same elevated negative potential and the plate is connected to the ground. Because the large difference between the curvatures radii of the two elements, the corona discharge takes place only at the surface of the wire (i.e. ionizing electrode).

According to the model adopted for the present study, the ions leave the wire surface and move toward the grounded plate with constant mobility. The electrodes are placed in air such that $\vec{D}=\varepsilon_{0} \vec{E}, \vec{D}$ and $\vec{E}$ being the electric displacement and field strength, respectively, and $\varepsilon_{0}$ the vacuum permittivity.

\subsection{Governing equations}

The electric field $\vec{E}$ affected by the presence of the negative ions is governed by the following equations:

- the Poisson equation:

$$
\Delta V=-\frac{\rho}{\varepsilon_{0}},
$$

where $\rho$ is the ionic space charge density and $V$ is the electric potential related to the electric field through

$$
\vec{E}=-\operatorname{grad} V \text {. }
$$

- the charge conservation law:

$$
\frac{\partial \rho}{\partial t}+\operatorname{div} \vec{j}=0,
$$

where $\vec{j}=\rho K_{i} \vec{E}$ is the corona current density when the diffusion current is neglected. $K_{\mathrm{i}}=2.410^{-4} \mathrm{~m}^{2} / \mathrm{Vs}$ being the ion mobility [11].

\subsection{Computation domain}

Neglecting the edge effects of the dual electrode and considering that the corona discharge is constant and uniform all along each cylindrical wire generating line, the coupled electric field and space charge problem has plane symmetry. The bi-dimensional computation domain is obtained by taking the cross-section $(O x y)$ of the electrodes system (see Fig. 1). Because the symmetry of the electrodes, it is possible to consider only the plane limited by the $O x$ axis (where $y \geqslant 0$ ). A more detailed presentation is given in $[11,12]$. 


\subsection{Boundary conditions}

The boundary conditions are also discussed in [11]. For the electrical potential (Poisson equation (1)), the boundary conditions are of Dirichlet type for the wire and the non-ionizing electrode $\left(V=V_{0}\right.$, where $V_{0}$ is the applied potential) as well as for the grounded collector plate $(V=0)$. On the symmetry axis $O x$, a Neumann type condition is imposed $(\partial V / \partial y=0)$.

For the charge conservation equation (3), the boundary condition consists in imposing a charge density value in all considerate injection points $Q$ located all around the wire surface $\rho_{0}(Q)$.

The method that permits to establish the value of $\rho_{0}(Q)$ in each $Q$ injection point is based on the Kaptzov hypothesis and Peek relation [11]:

$E_{\mathrm{P}}=31 \cdot \delta_{\mathrm{R}}\left(1+\frac{0.308}{\sqrt{r}}\right)$,

where $E_{\mathrm{P}}$ is the threshold field in $\mathrm{kV} / \mathrm{cm}, \delta_{\mathrm{R}}=3.92 \cdot p / T$, with $p$ the air pressure in bar, $T$ the thermodynamic temperature in $\mathrm{K}$ and $r$ is the radius of the wire in $\mathrm{cm}$.

Therefore, the boundary condition for the charge conservation equation (3) is replaced by the additional condition for the field strength at the surface of the ionizing wire [11]: the electric field $E$ in a point $Q$ at the surface of the ionizing electrode remains constant and equals the Peek field when voltage is increased beyond the corona inception level, i.e. $E(Q)=E_{\mathrm{P}}$.

In the previous papers [11,12], the computation algorithm was simplified by assuming that the charge injection is uniform all around the wire circumference. In this way the charge density had the same value $\rho_{0}$ in all injecting points $Q$. As a consequence of Kaptzov's hypothesis adopted above, the charge boundary condition had the form $\langle E\rangle=E_{\mathrm{p}}$, where $\langle E\rangle$ was the mean value of the field strength at the surface of the wire. This approach disregarded the modulation of the electric field at the surface of the ionizing electrode and considered that the corona discharge had the same magnitude in any point at the wire surface.

The present paper refines the boundary condition of the charge conservation equation by taking into account the variation of electric field strength on the surface of the wire due to the presence of the cylindrical support to which the ionizing element is attached. This time the condition $E(Q)=E_{\mathrm{P}}$ will be treated distinct for any point $Q$, so that to simulate a corona discharge with a magnitude that varies with the position on the wire surface. As a consequence, the value of the charge density on the cathode surface will be dependent of the position of the point $Q$. The basic assumption of this work still remains the validity of Peek's formula (as established for axial wire-cylinder configuration). A point located on the ionizing wire will be considerate an "injector of charge" only if the local field strength is higher than $E_{\mathrm{P}}$.

\subsection{Computation method}

The computation method used to find the solution of the system (1)-(3) is detailed in [11]. A conformal mapping transforms the computational domain into another one, on which the Poisson and charge conservation equations are much easier to solve, using classical numerical techniques: finite differences method (FDM) to solve (1) and (2) and the method of characteristics (MOC) to solve (3). Both problems are solved iteratively until the solution of the system of equations is reached (the values of $V, E, \rho$ and $\rho_{0}(Q)$ are obtained for all the points of the domain).

\section{Modulation of charge injection on the wire surface}

The corona discharge around the cathode wire depends on the local variation of the electric field. For a cylindrical wire located far away from another electrode, the field strength on its surface is constant and, by consequence, the corona discharge is uniform. Considering the wire-plate geometry with a reasonable $d$ inter-electrode distance (in the cm-range), the influence of a grounded plate on the wire circumference field is perceptible; the field increases non-uniformly on the wire circumference and become higher in the region facing the plate (see Fig. 2). The maximum variation between the $E(\mathrm{~A})$ and $E(\mathrm{~B})$ - see Fig. 1 -is $1.54 \%$ for $20 \mathrm{~mm}$ between the wire and plate and decrease at $0.62 \%$ if the gap increases at $50 \mathrm{~mm}$.

Theses results show that the hypothesis of an uniform charge injection all around of corona wire facing to a grounded plate can lead to a good precision of corona discharge simulation when the distance $d$ is higher than few centimeters.

The distortion of the electric field on the wire surface is more important in the case of the dual corona electrode (Fig. 1). There are two factors that influence the field and injection modulation: the proximity of the grounded plate

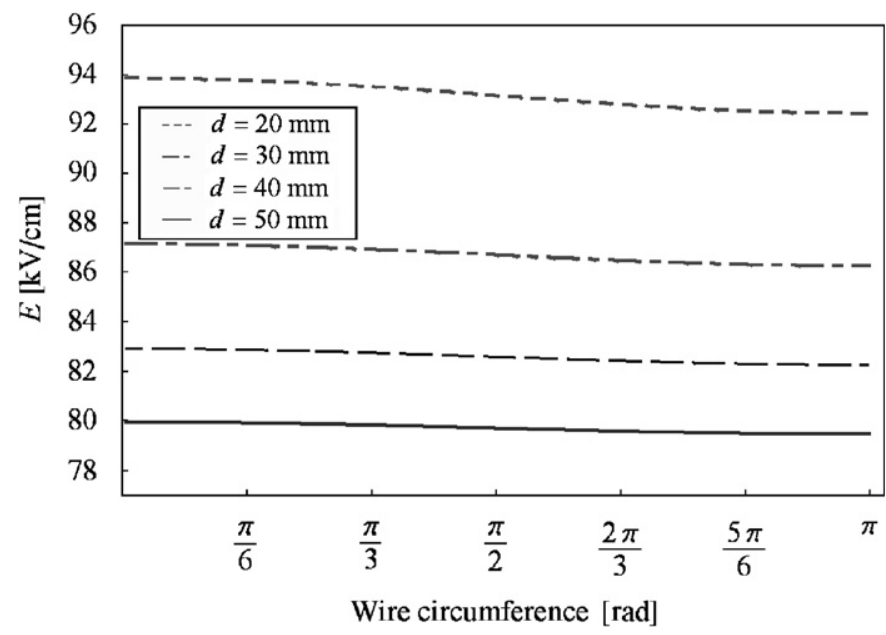

Fig. 2. Variation of electrostatic field strength around the ionizing wire for wire-plate electrode configuration $\left(V_{0}=8 \mathrm{kV}, R=13.4 \mathrm{~mm}\right.$, $h=20 \mathrm{~mm}$, and $r=0.1 \mathrm{~mm}$ ). 


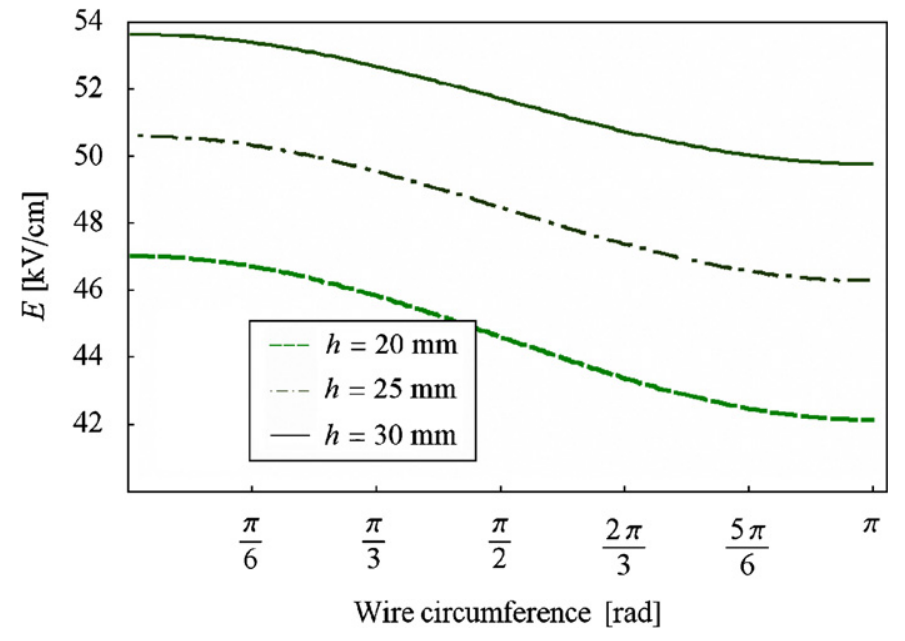

Fig. 3. Variation of electrostatic field strength around the ionizing wire for cylinder-wire-plate electrode configuration $\left(V_{0}=8 \mathrm{kV}, R=13.4 \mathrm{~mm}\right.$, $d=40 \mathrm{~mm}$, and $r=0.1 \mathrm{~mm}$ ).

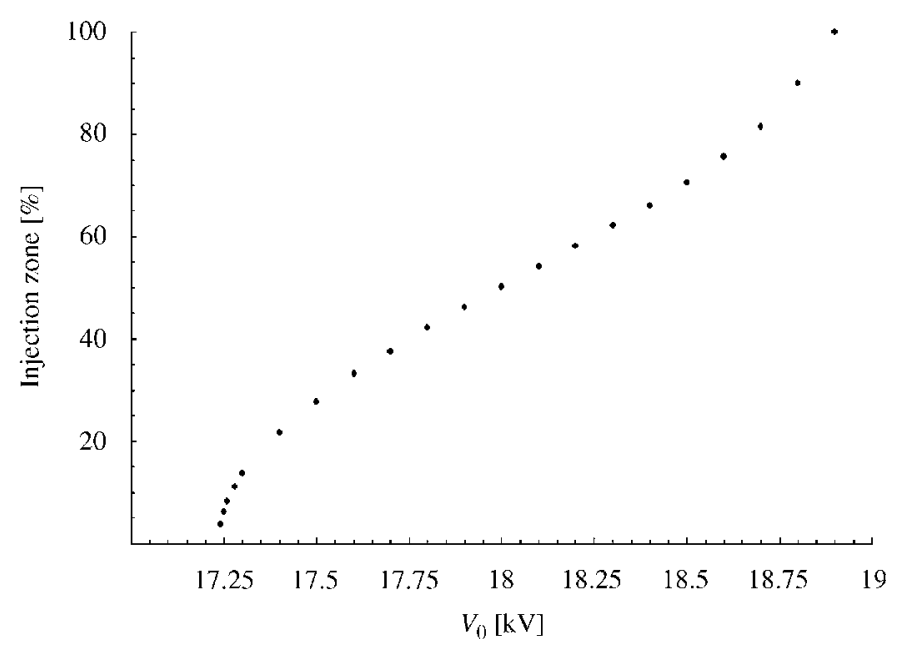

Fig. 4. Corona discharge zone versus electrical potential (wire-plate distance $d=40 \mathrm{~mm}$ and dual cylinder-wire space $h=25 \mathrm{~mm}, r=0.1 \mathrm{~mm}$, and $R=13.4 \mathrm{~mm}$ ).

and the distance to the dual cylinder. Fig. 3 presents the variation of electrostatic field on the wire circumference and shows clearly the impact of the presence of the dual cylinder. The field strongly decreases at the wire surface (only $55 \%$ of wire-plate field strength for wire-cylinder space $h=20 \mathrm{~mm}$ ) and its modulation becomes very important. For a wire-plate gap equal to $40 \mathrm{~mm}$, the maximum variation of wire surface field is $7.8 \%$ for $h=30 \mathrm{~mm}$ and increase at $11.6 \%$ for $h=20 \mathrm{~mm}$. This important electric field modulation on the wire surface cannot be neglected and should be reflected in a nonuniform corona discharge model.

In accord with the non-uniformity of the electric field variation on the wire surface Fig. 4 shows the extension of the corona circumference zone (where the field strength at the wire surface is higher than $E_{\mathrm{p}}$ ). For the given wire radius the injecting region of the wire depends on the applied voltage and only for $V_{0}=19 \mathrm{kV}$ the entire circumference injects the negative ions.

Based on theses numerical results, the boundary condition of the charge conservation equation will be adapted by a local treatment of the relation $E(Q)=E_{\mathrm{P}}$ and the computation of the field-charge distributions will be refined.

\section{Field-charge computation for non-uniform corona discharge}

Using the same numerical algorithm described in Section 2.5 and detailed in [11,12], and considering a non-uniform

\section{a}

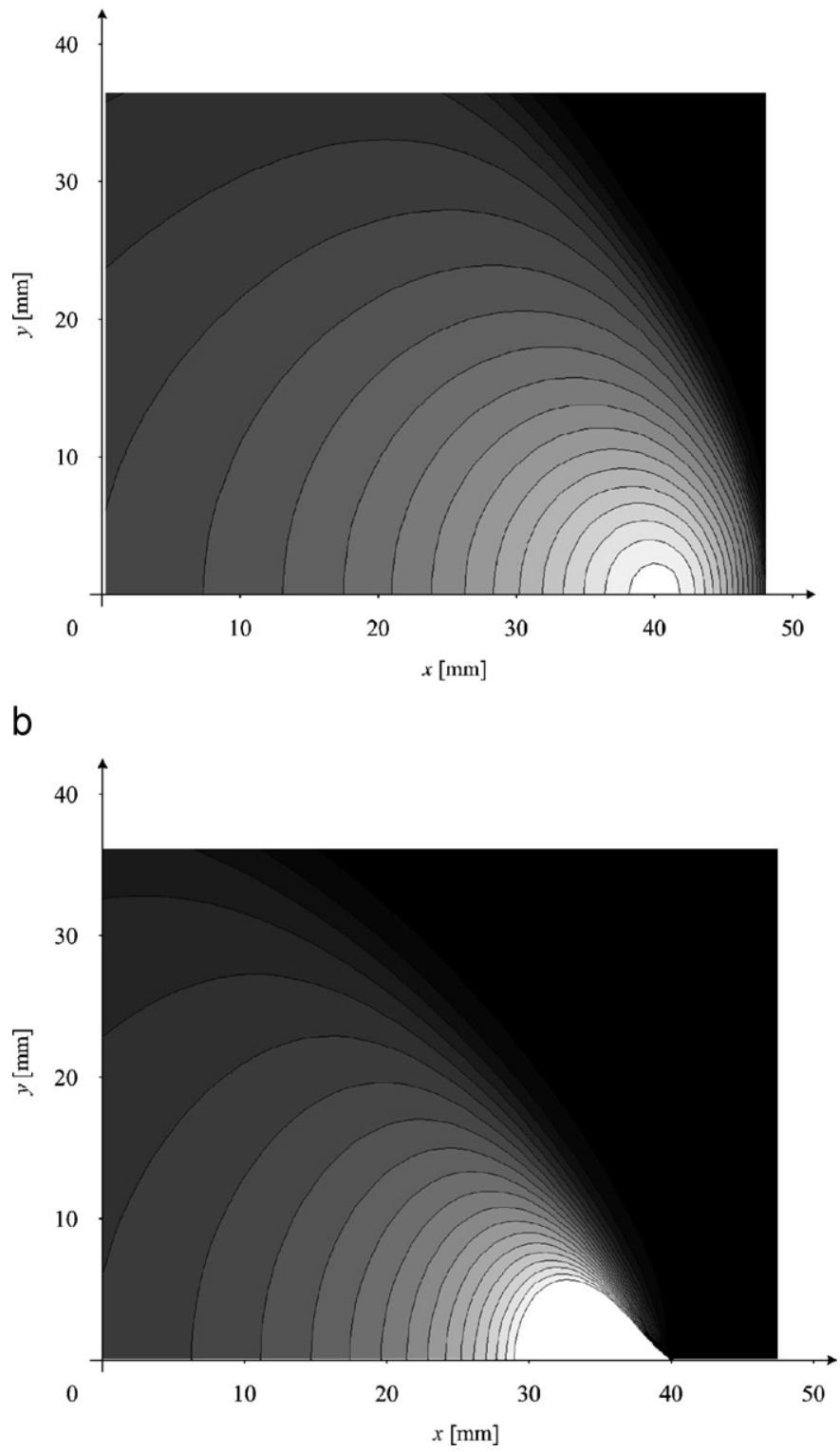

Fig. 5. Space charge distribution (iso-charge lines) near the ionizing wire (for $h=20 \mathrm{~mm}, d=40 \mathrm{~mm}, r=0.1 \mathrm{~mm}, R=13.4 \mathrm{~mm}$, and $V_{0}=30 \mathrm{kV}$ ) (a) for a supposed uniform corona discharge around the wire circumference and (b) for a non-uniform charge injection around the wire circumference (on the cathode surface). 
injection of charge on the circumference of the ionizing wire, the spatial distributions of the electric field and space charge are refined. For each computation step, the injection of charge is controlled in any point considered on the wire circumference and is adapted until the computed field on the wire surface is equal to $E_{\mathrm{P}}$.

The resulting charge density values on the wire surface are related to the variation of the electric field strength and decrease from the left symmetry point A to the point B-see Fig. 1. When the applied voltage is lower, the right face of the wire is not active and the resulting charge density in this zone tends to zero. However, for very high values of the applied voltage, the wire injects in all points. As expected, the maximum value of charge density corresponds to the symmetry point $\mathrm{A}$. The rate of fall of $\rho_{0}^{*}$ toward point B is dependent on the magnitude of applied voltage.

Fig. 5b shows clearly the important change of the spatial distribution of the ionic charge produced by the nonuniform injection of the cathode, compared to the uniform corona discharge case (Fig. 5a). For the case shown in Fig. 5, the injection takes place only on the left side of the wire and the region affected by the presence of the spatial charge is more restricted in the $O y$ direction.

Fig. 6 displays the variation of the electric field at the surface of the grounded plate as a function of the $y$ coordinate. The different injection laws at the cathode modify the electric field only in the central zone of the plate as effect of the changed ionic space charge distribution. For the non-uniform injection, the higher ion density in the central zone increases the field strength by $10 \%$ as compared to the case of uniform discharge.

The difference between the two situations is more important in the case of the ionic charge density and current distribution on the surface of the grounded plate (Figs. 7 and 8). At the center of the plate (i.e. region closest to the wire), the current and space charge densities for non-uniform injection case are higher by about $20 \%$.

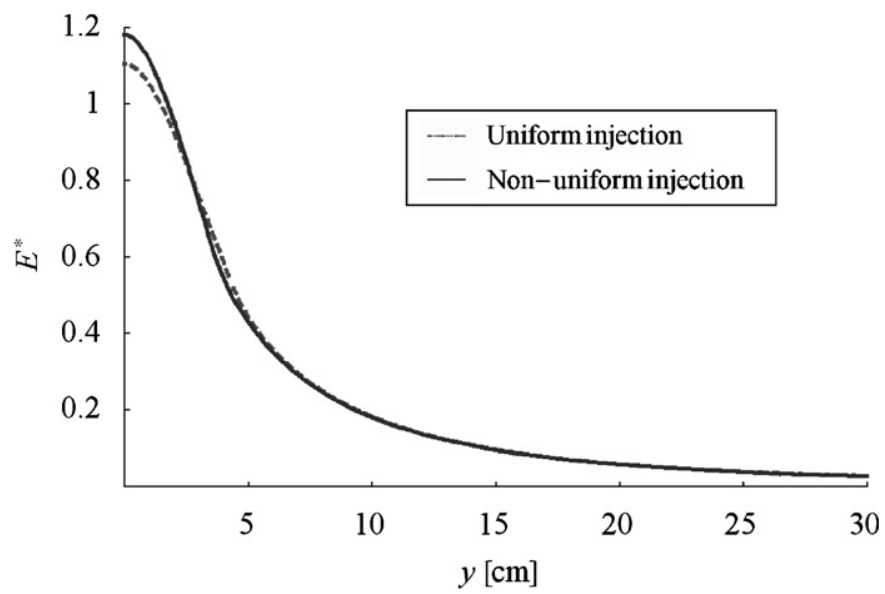

Fig. 6. Variation of normalized field strength along $y$ coordinate on the grounded plate for uniform and non-uniform charge injection ( $h=20 \mathrm{~mm}, d=40 \mathrm{~mm}, r=0.1 \mathrm{~mm}, R=13.4 \mathrm{~mm}, V_{0}=30 \mathrm{kV}$, and the reference field $E_{\text {ref }}=V_{0} / d=0.75 \mathrm{kV} / \mathrm{mm}$ ).

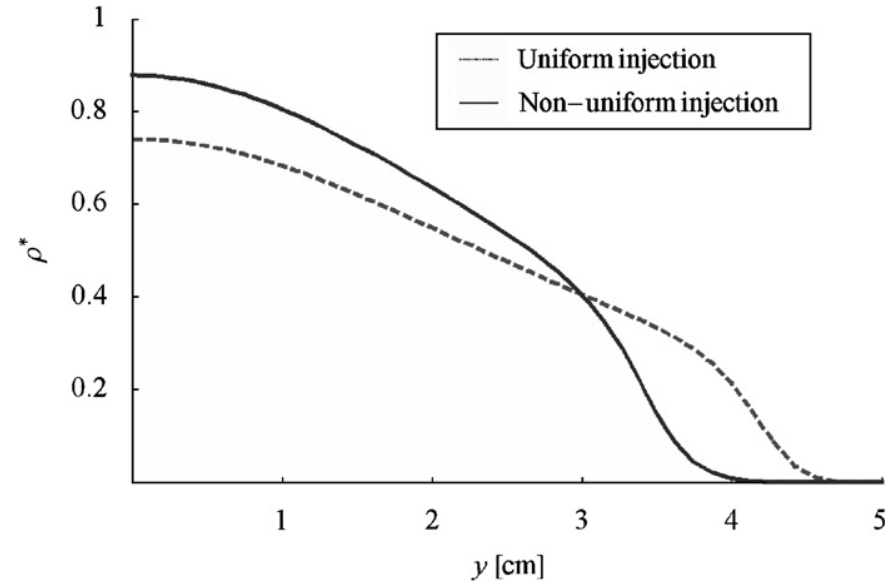

Fig. 7. Variation of normalized charge density along $y$ coordinate on the grounded plate for uniform and non-uniform charge injection ( $h=20 \mathrm{~mm}, d=40 \mathrm{~mm}, r=0.1 \mathrm{~mm}, R=13.4 \mathrm{~mm}, V_{0}=30 \mathrm{kV}$, and the reference charge density $\rho_{\text {ref }}=\varepsilon_{0} V_{0} / d^{2}=6.46 \mu \mathrm{C} / \mathrm{m}^{3}$ ).

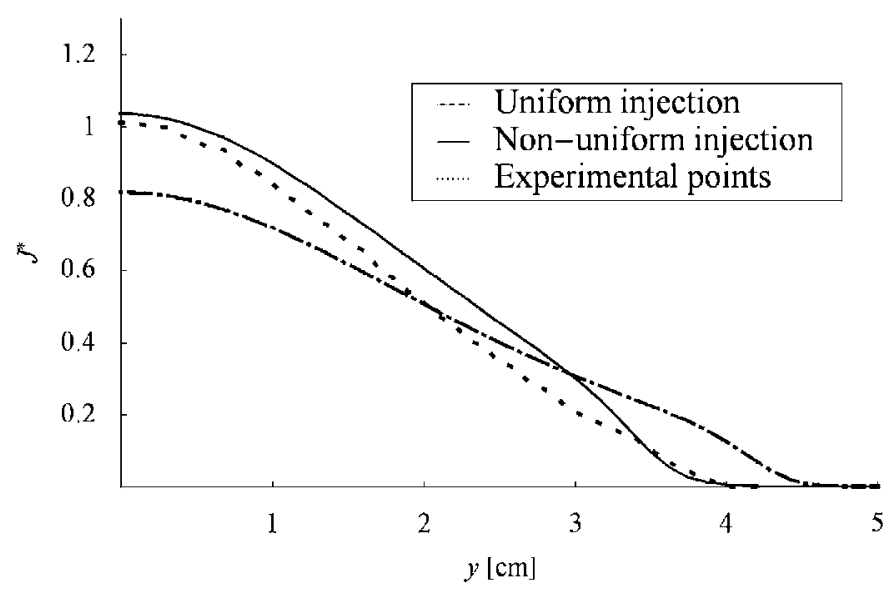

Fig. 8. Variation of normalized computed and experimental ionic current density along $y$ coordinate on the grounded plate for uniform and nonuniform charge injection $(h=20 \mathrm{~mm}, d=40 \mathrm{~mm}, r=0.1 \mathrm{~mm}$, $R=13.4 \mathrm{~mm}, V_{0}=30 \mathrm{kV}$ and the reference current density $j_{\text {ref }}=K$ $\left.\varepsilon_{0} V_{0}^{2} / d^{3}=29.87 \mathrm{~mA} / \mathrm{m}^{2}\right)$.

However, the spatial extension of theses quantities along $y$ direction becomes smaller.

In accord with the experimental measurements (Fig. 8) obtained with the set-up described in $[11,12]$ the numerical algorithm that considers the modulation of the electric field on the wire surface offers more accurate results. The values of the current density in the central zone are very close to the experimental data and the extension of the grounded plate zone that receives the ionic current is identical to the experimental one.

\section{Conclusions}

The paper is focused on modelling the charge injection phenomena at the surface of the ionizing wire of a dual corona electrode. The computation algorithm based on the FDM and MOC techniques that was detailed in two 
previous papers has been refined so as to incorporate more realistic boundary conditions for the charge conservation equation. Thus, the new algorithm takes into account the modulation of the electric field at the wire surface induced by the presence of the dual high voltage cylinder and the grounded plate, preserving at the same time the validity of Peek's formula. Compared to the uniform corona discharge case, the numerical results reported in the paper point out important changes, especially for the space charge distribution and the ionic current distribution at the surface of the collecting plate electrode.

The changes made to the initial algorithm preserve the stability of this technique, the convergence rate of which depends on the applied voltage. For small values of $V_{0}$ (close to the inception corona voltage) a few iterations are sufficient to find the solution. However, for high values of the potential, the number of iterations becomes important. For small (less than $1 \mathrm{~cm}$ ) wire-cylinder distances, the algorithm failed because Kaptzov's condition is no longer satisfied.

\section{Acknowledgment}

The authors would like to thank Octavian Blejan for helping with the experimental measurements.

\section{References}

[1] A.D. Moore (Ed.), Electrostatics and Its Applications, Wiley, New York, 1973.
[2] J.S. Chang, A.J. Kelly, J.M. Crowley (Eds), Handbook of Electrostatic Processes, Dekker, New York, 1995.

[3] K.R. Parker, Electrostatic Precipitation, Chapman \& Hall, New York, 1997.

[4] R.S. Sigmond, The unipolar corona space charge flow problem, J. Electrostatics 18 (1986) 249-272.

[5] P. Atten, Méthode générale de résolution du problème du champ électrique modifié par une charge d'espace unipolaire injectée, Rev. Gén. Electr. 83 (1974) 143-153.

[6] B.S. Rajanikanth, B.R. Prabhakar, Modeling of prebreakdown VI characteristics of a wire-plate electrostatic precipitator operating under combined dc-pulse energization, IEEE. Trans. Dielectr. Electr. Insul. 1 (1994) 1058-1067.

[7] V. Jaiswal, M.J. Thomas, Finite element modelling of ionized field quantities around a monopolar HVDC transmission lines, J. Phys. D Appl. Phys. 36 (2003) 3089-3094.

[8] K. Adamiak, V. Atrazhev, P. Atten, Corona discharge in the hyperbolic point-plane configuration: direct ionization criterion versus approximate formulation, IEEE Trans. Dielectr. Electr. Insul. 12 (2005) 1025-1033.

[9] J. Davis, J. Hoburg, Wire-Duct precipitator field and charge computation using finite element and characteristics methods, J. Electrostatics 14 (1983) 187-199.

[10] A.A. Elmoursi, G.S.P. Castle, Modelling of corona characteristics in a wire-duct precipitator using the charge simulation technique, IEEE Trans. Ind. Appl. 23 (1987) 95-102.

[11] L.M. Dumitran, P. Atten, P.V. Notingher, L. Dascalescu, 2-D corona field computation in configurations with ionising and non-ionising electrodes, J. Electrostatics 64 (2006) 176-186.

[12] L.M. Dumitran, L. Dascalescu, P. Atten, P.V. Noţingher, Computational and experimental study of ionic space charge generated by combined corona-electrostatic electrode systems, IEEE Trans. Ind. Appl. 42 (2006) 378-384. 